\title{
Late-Onset Langerhans Cell Histiocytosis with Cerebellar Ataxia as an Initial Symptom
}

\author{
Jung-Min Pyun ${ }^{\mathrm{a}}$ Hyeyoung Park ${ }^{\mathrm{b}}$ Kyung Chul Moon ${ }^{\mathrm{c}}$ Beomseok Jeon ${ }^{\mathrm{a}}$ \\ ${ }^{a}$ Department of Neurology, Seoul National University Hospital, College of Medicine,

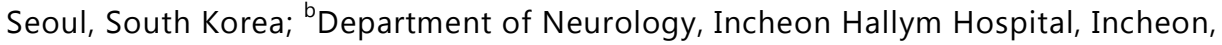 \\ South Korea; 'Department of Pathology, Seoul National University Hospital, College of \\ Medicine, Seoul, South Korea
}

\section{Keywords}

Cerebellar ataxia $\cdot$ Langerhans cell histiocytosis

\begin{abstract}
Late-onset progressive cerebellar ataxia is a diagnostic challenge because of a poor correlation between genotype and phenotype, and a broad range of secondary causes that extend beyond the neurological field. We report the case of a 45-year-old woman admitted after 2 years of slowly progressing cerebellar ataxia, dysarthria, and emotional instability. Notably, she was diagnosed with diabetes insipidus at the age of 35. As 'idiopathic cerebellar ataxia' was suspected, diagnostic tests, including genetic testing as well as serum and cerebrospinal fluid analyses, and brain magnetic resonance imaging (MRI) were performed. All results were normal except those of MRI, performed 9 months prior to admission, which showed multiple dot-like white matter lesions with unclear cause. On a repeated brain MRI, a new lesion presenting as a 1.5-cm-sized highly enhancing mass attached to the right frontal skull was found. A sharply marginated lytic skull defect was also evident on skull X-ray, which corresponded to the lesion mass. Given these new radiological findings, a systemic review of the patient's medical history for rare secondary causes of cerebellar ataxia was performed, with particular attention to her past 'diabetes insipidus'. The mass, lytic lesion of the skull, white matter lesion, diabetes insipidus, and cerebellar ataxia all suggested a final diagnosis of Langerhans cell histiocytosis (LCH), which was confirmed histopathologically. This is a rare case
\end{abstract}


of late-onset LCH with an unusual initial symptom which underlines the importance of carefully reviewing the patient's medical history and broadening the search for etiologies beyond the nervous system.

\section{Introduction}

The initial diagnostic steps for a neurological problem comprise an evaluation of the patient's current medical status and medical history, as well as an evaluation of neurological deficits. Various differential diagnoses are initially suggested by the localization of a potential lesions and the time course of symptom development, and several tests are then performed to reach a final diagnosis. Selecting significant findings among the clinical information may be difficult, as the information may seem irrelevant to differential diagnoses with a high incidence. We report on the case of a patient with cerebellar ataxia whose medical history provided elements that led to an unusual and interesting final diagnosis.

Langerhans cell histiocytosis (LCH) is a rare proliferative disorder of Langerhans cells, which are antigen-presenting dendritic cells $[1,2]$. Whether LCH is an immunologic reactive disorder or a myeloproliferative neoplastic disorder has not been clearly elucidated $[3,4]$. This disease most often affects young children; however, onset during adulthood has also been reported. The incidence of childhood LCH is 1:200,000 and peaks between 1 and 4 years of age [5]. Adulthood LCH has been reported at an incidence of 1:1,000,000, and the mean age at presentation is 34 years [5]. LCH can manifest in one or many body systems or tissues [6].

The purpose of this paper is to report a rare case of LCH with late-onset cerebellar ataxia as an initial symptom. The patient's past medical history and the finding of a skull lesion led to an unusual and interesting final diagnosis.

\section{Case Report}

A 45-year-old female was referred to our clinic with a 2-year history of gait disturbance and imbalance. Her veering tendency worsened progressively, resulting in frequent falls, and her speech was becoming dysarthric. In addition, she became emotionally unstable and could not control her laughter. Her past medical history revealed diabetes insipidus, which had been diagnosed at the age of 35 and treated with desmopressin nasal spray. She was referred to us with a diagnosis of 'idiopathic adult-onset cerebellar ataxia'.

On examination, hypermetric saccade, cogwheel smooth pursuit, and dysarthria with nasal sound were noted. Finger-to-nose and heel-to-shin tests revealed bilateral limb ataxia. The patient exhibited ataxic gait with a wide base and poor tandem gait. Her cognitive function was intact as assessed by Mini-Mental State Examination (30/30). Motor power, muscle tone, sensation, and deep tendon reflexes were normal.

Laboratory examinations showed normal levels of serum rapid plasma reagin, vitamin E, and alpha-fetoprotein. The results of genetic tests for spinocerebellar ataxia type 1, 2, 3, 6, 7, and 17, dentatorubropallidoluysian atrophy, and Friedreich ataxia were normal. The result of cerebrospinal fluid analysis was also normal.

Magnetic resonance imaging (MRI) of the patient's brain performed in another hospital 9 months prior to admission to our clinic showed cerebellar atrophy with pituitary stalk thickening (fig. 1a) and dot-like white matter changes in both hemispheres (fig. 1b). Repeat- 
Pyun et al.: Late-Onset Langerhans Cell Histiocytosis with Cerebellar Ataxia as an Initial Symptom

ed brain MRI scans in our hospital showed no interval changes of the finding described above. However, one new lesion presenting as a 1.5 -cm-sized highly enhancing mass attached to the right frontal skull was found (fig. 1c). The mass corresponded to a sharply marginated lytic skull defect in the right frontal bone on the skull X-ray (fig. 1d).

An excisional biopsy of the mass showed atypical histiocytic collections with eosinophil infiltration on hematoxylin and eosin staining. Immunohistochemical analysis revealed CD1a-, JL-1-, and langerin-positive cells, which was consistent with a diagnosis of LCH (fig. 2).

A computed tomography scan of the neck, chest, abdomen, and pelvis showed an osteolytic lesion with peripheral osteosclerosis in the left sacrum. Bone marrow biopsy results were normal.

The patient was treated with vinblastine $10 \mathrm{mg}\left(6 \mathrm{mg} / \mathrm{m}^{2}\right)$ and prednisolone $65 \mathrm{mg}$ $\left(40 \mathrm{mg} / \mathrm{m}^{2}\right)$. A follow-up using brain MRI 3 months after chemotherapy showed disappearance of the enhancing mass in the right frontal bone and pituitary stalk thickening. Clinically, however, there was slow progression of the symptoms, leading to a wheelchair-bound state within 2 years.

\section{Discussion}

Cerebellar atrophy evidenced by brain MRI performed in another hospital was compatible with the patient's clinical diagnosis of cerebellar ataxia. However, her past medical history of 'diabetes insipidus' with pituitary stalk thickening and multiple dot-like white matter lesions should have suggested a secondary cause. This is especially true because this 45 year-old patient had no risk factors for small vessel disease. The findings of a new mass lesion on MRI, the lytic defect on the skull X-ray image, and the subsequent histopathological examination of the mass led to a diagnosis of $\mathrm{LCH}$.

Late-onset progressive cerebellar ataxia is a diagnostic challenge. The correlation between genotype and phenotype is poor, and various secondary causes of cerebellar ataxia beyond the neurological field exist. In our case, the patient's past medical history of 'diabetes insipidus' became an important clue.

It was hard to suspect LCH exclusively from the patient's clinical symptoms, given that cerebellar ataxia is not a typical initial symptom of LCH and that its onset was late for LCH. According to a study from the Mayo Clinic that included 314 patients diagnosed with LCH over a period of 50 years, the dominant presenting features were local bone pain (41\%), dyspnea (14\%), malaise or abnormal chest X-ray (9\%), painful scalp lump (7\%), diabetes insipidus (5\%), head and neck lymphadenopathy (2.2\%), and so on [6]. A more recent study from the Histiocyte Society was conducted on 274 adult patients with LCH. The data from that study showed that the patients had symptoms in the bones (38.8\%), lungs (31.7\%), skin (15.4\%), and lymph nodes (6.3\%), and were diagnosed with diabetes insipidus (29.6\%) [5]. The most common central nervous system involvement in LCH is infiltration of the hypothalamic pituitary region by LCH granuloma, frequently leading to diabetes insipidus and anterior pituitary hormone deficiency [7].

LCH patients presenting with cerebellar ataxia have been rarely reported, and even then exclusively pediatric cases [8-12]. In those patients, cerebellar ataxia appeared with LCH progression after initial presentation of a skin or bone lesion.

To our knowledge, a case of initial cerebellar ataxia in an adult had not been reported previously. Our case underlines the importance of carefully reviewing the patient's medical 
Pyun et al.: Late-Onset Langerhans Cell Histiocytosis with Cerebellar Ataxia as an Initial Symptom

history and broadening the search for causes beyond the nervous system in the differential diagnosis of cerebellar ataxia.

\section{Statement of Ethics}

Informed consent was obtained from the patient.

\section{Disclosure Statement}

The authors have no conflicts of interest to disclose.

\section{References}

1 Satter EK, High WA: Langerhans cell histiocytosis: a review of the current recommendations of the Histiocyte Society. Pediatr Dermatol 2008;25:291-295.

2 Yu RC, Chu C, Buluwela L, et al: Clonal proliferation of Langerhans cells in Langerhans cell histiocytosis. Lancet 1994;343:767-768.

-3 Morimoto A, Oh Y, Shioda Y, et al: Recent advances in Langerhans cell histiocytosis. Pediatr Int 2014;56: 451-461.

4 Abla 0, Egeler RM, Weitzman S: Langerhans cell histiocytosis: current concepts and treatments. Cancer Treat Rev 2010;36:354-359.

5 Arico M, Girschikofsky M, Genereau T, et al: Langerhans cell histiocytosis in adults. Report from the International Registry of the Histiocyte Society. Eur J Cancer 2003;39:2341-2348.

-6 Howarth DM, Gilchrist GS, Mullan BP, et al: Langerhans cell histiocytosis: diagnosis, natural history, management, and outcome. Cancer 1999;85:2278-2290.

7 Rois N, Prayer D, Prosch H, et al: Neuropathology of CNS disease in Langerhans cell histiocytosis. Brain 2005;128:829-838.

-8 Imashuku S, Ishida S, Koike K, et al: Cerebellar ataxia in pediatric patients with Langerhans cell histiocytosis. J Pediatr Hematol Oncol 2004;26:735-739.

-9 Nakamura T, Morimoto N, Goto F, et al: Langerhans cell histiocytosis with disequilibrium. Auris Nasus Larynx 2012;39:627-630.

10 Poe LB, Dubowy RL, Hochhauser L, et al: Demyelinating and gliotic cerebellar lesions in Langerhans cell histiocytosis. AJNR Am J Neuroradiol 1994;15:1921-1928.

11 Goldberg-Stern H, Weitz R, Zaizov R, et al: Progressive spinocerebellar degeneration 'plus' associated with Langerhans cell histiocytosis: a new paraneoplastic syndrome? J Neurol Neurosurg Psychiatry 1995;58:180-183.

12 Shuper A, Stark B, Yaniv Y, et al: Cerebellar involvement in Langerhans' cell histiocytosis: a progressive neuropsychiatric disease. J Child Neurol 2000;15:824-826. 


\section{Case Reports in Neurology}

\begin{tabular}{l|l}
\hline Case Rep Neurol 2016;8:218-223 \\
\hline DOI: 10.1159/000450884 & $\begin{array}{l}\text { C 2016 The Author(s). Published by S. Karger AG, Basel } \\
\text { www.karger.com/crn }\end{array}$ \\
\hline
\end{tabular}

Pyun et al.: Late-Onset Langerhans Cell Histiocytosis with Cerebellar Ataxia as an Initial Symptom

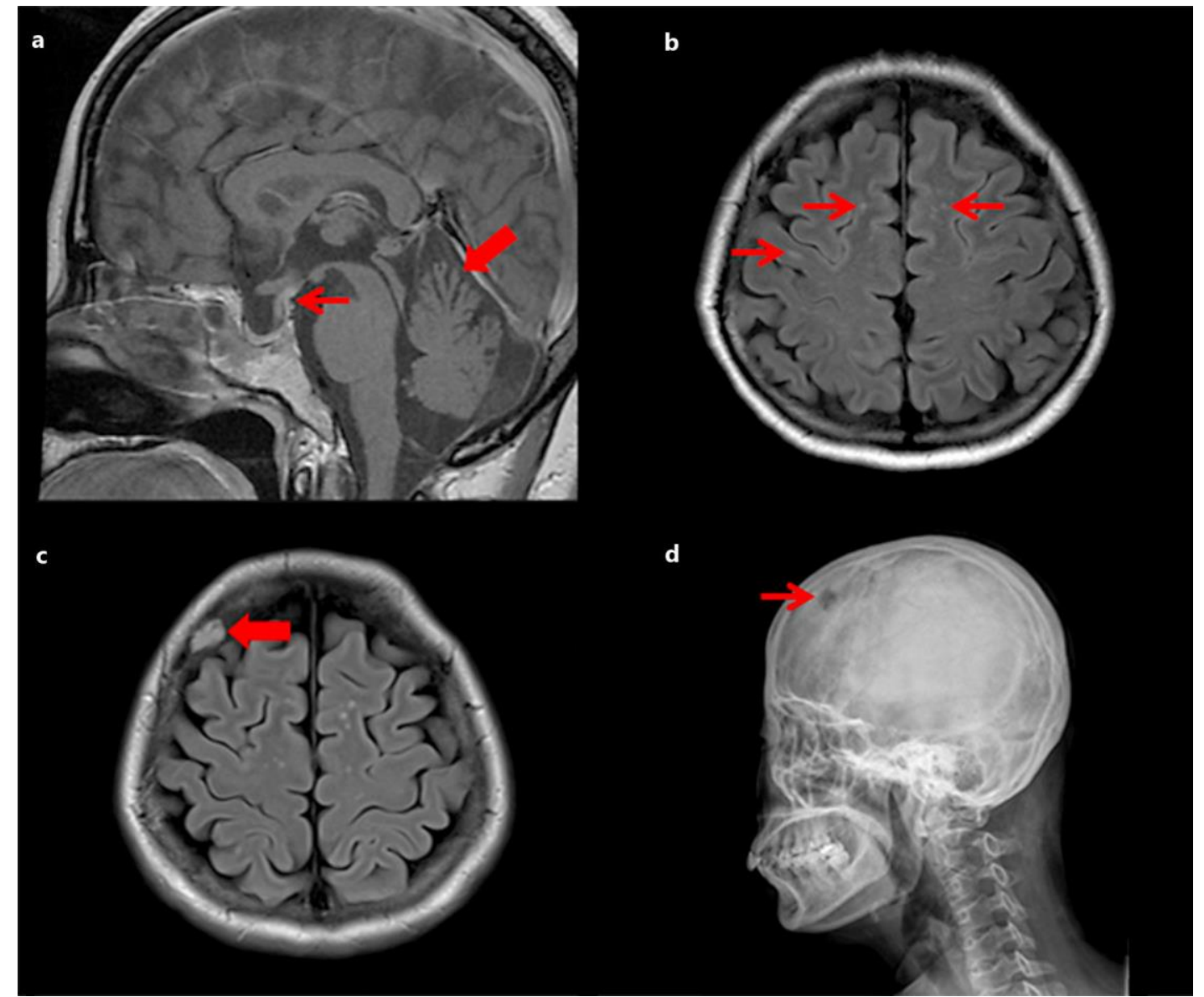

Fig. 1. a Cerebellar atrophy (thick arrow) and pituitary stalk thickening (thin arrow). b Dot-like white matter changes (arrows). c MRI image 9 months after the previous MRI shows a new mass lesion on the right frontal skull (arrow) corresponding to an osteolytic lesion in the right frontal skull (d). 


\section{Case Reports in Neurology}

www.karger.com/crn

Pyun et al.: Late-Onset Langerhans Cell Histiocytosis with Cerebellar Ataxia as an Initial Symptom

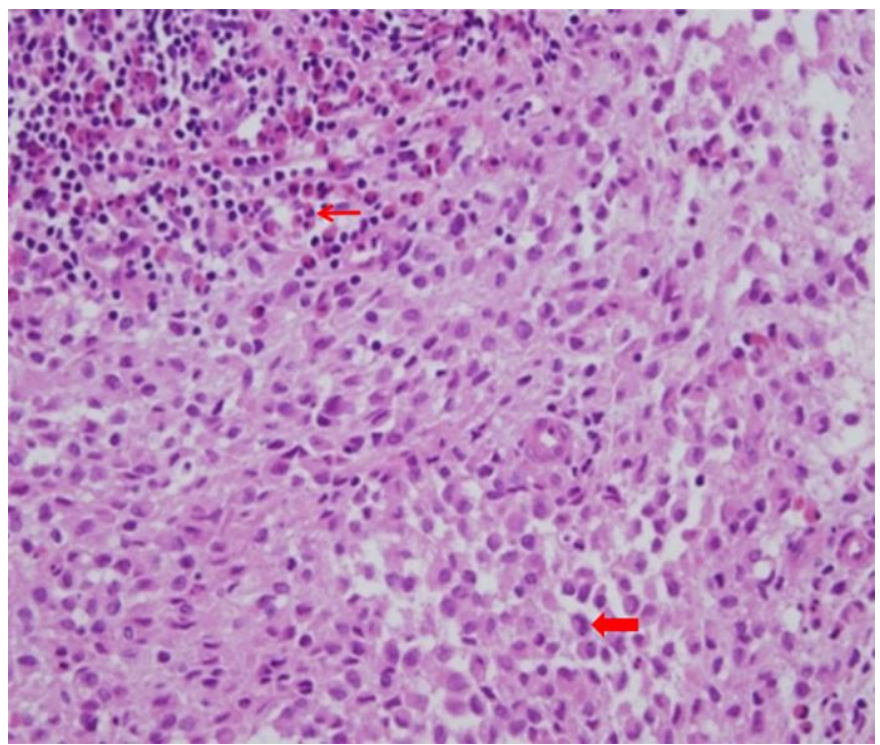

Fig. 2. Langerhans cells (thick arrow) having 'coffee-bean'-shaped grooved nuclei are admixed with eosinophils (thin arrow). HE staining, $\times 400$. 